\title{
Investigation of statistical characteristics of interaction between the low-frequency oscillations in heart rate variability and photoplethysmographic waveform variability in healthy subjects and myocardial infarction patients
}

\author{
Vladimir A. Shvartz ${ }^{1}$, Anatoly S. Karavaev ${ }^{2}$, Ekaterina I. Borovkova ${ }^{2}$, Sergey A. Mironov ${ }^{3}$, \\ Vladimir I. Ponomarenko ${ }^{2,4}$, Mikhail D. Prokhorov ${ }^{4}$, Yurii M. Ishbulatov ${ }^{2}$, \\ Elena E. Lapsheva ${ }^{2}$, Vladimir I. Gridnev ${ }^{2,5}$, Anton R. Kiselev ${ }^{1,2,5}$ \\ ${ }^{1}$ Bakulev Scientific Center for Cardiovascular Surgery, Moscow, Russia \\ ${ }^{2}$ Saratov State University n.a. N.G. Chernyshevsky, Saratov, Russia \\ ${ }^{3}$ Central Clinical Military Hospital, Moscow, Russia \\ ${ }^{4}$ Saratov Branch of the Institute of Radio Engineering and Electronics of Russian Academy of Sciences, Saratov, Russia \\ ${ }^{5}$ Saratov State Medical University n.a. V.I. Razumovsky, Saratov, Russia
}

Received 15 January 2016, Accepted 21 March 2016

Original Text in Russian: (C) Shvartz V.A., Karavaev A.S., Borovkova E.I., Mironov S.A., Ponomarenko V.I., Prokhorov M.D., Butenko A.A., Gridnev V.I., Kiselev A.R., 2015, published in Saratov Journal of Medical Scientific Research 2015; 11(4): 537-542.

English version: (C) 2016, Shvartz V.A., Karavaev A.S., Borovkova E.I., Mironov S.A., Ponomarenko V.I., Prokhorov M.D., Ishbulatov Yu.M., Lapsheva E.E., Gridnev V.I., Kiselev A.R.

English version: (C) 2016, Russian Open Medical Journal

Abstract: Objective - This study compares the statistical characteristics of interaction between $0.1 \mathrm{~Hz}$ oscillations in heart rate variability (HRV) and photoplethysmographic waveform variability (PPGV) in healthy subjects and patients with myocardial infarction (MI).

Material and Methods - We studied 23 healthy subjects ( 20 men and 3 women, aged $26 \pm 3$ years) and 23 patients ( 12 men and 11 women, aged $52 \pm 4$ years) at about one month after MI. The 10-minute signals of simultaneously recorded electrocardiogram (ECG) and photoplethysmogram (PPG) were studied. We calculated the total percentage of phase synchronization between the studied $0.1 \mathrm{~Hz}$ oscillations and estimated the distribution functions of duration of synchronous and non-synchronous epochs, the variability of basic frequency of oscillations, and variance of phase noises in $0.1 \mathrm{~Hz}$ oscillations in HRV and PPGV.

Results - The total percentage of phase synchronization between $0.1 \mathrm{~Hz}$ oscillations is significantly greater in healthy subjects than in MI patients $(47 \pm 3 \%$ and $26 \pm 4 \%$, respectively). Significant difference between these two groups in the distribution of duration of synchronous and non-synchronous epochs was not revealed. The MI patients had greater variance between the basic frequencies of $0.1 \mathrm{~Hz}$ oscillations in HRV and PPGV than healthy subjects. This phenomenon correlates with the increased level of phase noises in the records of MI patients. Conclusion - The quality of synchronization between $0.1 \mathrm{~Hz}$ oscillations in HRV and PPG is associated with the strength of influence of external factors (noises) and variability of the basic frequency of these oscillations.

Keywords: cardiovascular system, phase synchronization, time series, low-frequency oscillations

Cite as Shvartz VA, Karavaev AS, Borovkova El, Mironov SA, Ponomarenko VI, Prokhorov MD, Ishbulatov YM, Lapsheva EE, Gridnev VI, Kiselev AR. Investigation of statistical characteristics of interaction between the low-frequency oscillations in heart rate variability and photoplethysmographic waveform variability in healthy subjects and myocardial infarction patients. Russian Open Medical Journal 2016; 5: e0203.

Correspondence to Dr. Anton R. Kiselev. Address: Research Institute of Cardiology, 141, Chernyshevsky str., Saratov, 410028 , Russia. Phone: +7 (8452) 201 899. E-mail: kiselev@cardio-it.ru

\section{Introduction}

Today low-frequency oscillations in cardiovascular system draw high researcher's interest [1-3]. In particular, such oscillations with main frequency near $0.1 \mathrm{~Hz}$ can be detected in heart rate variability (HRV), blood pressure variability and peripheral blood flow variability. Perceptions about baroreflectory origin of $0.1 \mathrm{~Hz}$ oscillations are prevailing [4, 5]. There are evidence of functional independence between mechanisms of cardiovascular autonomic control that cause low-frequency oscillations in HRV and peripheral blood flow variability (assessed by photoplethysmographic waveform variability (PPGV))
$[6,7]$. Though, these mechanisms are actively interacting and, as we showed earlier [8], this interaction leads, in particular, to phase and frequency synchronization. However, synchronization between $0.1 \mathrm{~Hz}$ oscillations in HRV and PPGV is not permanent even for healthy subjects. This led to the development of quantitative measure of synchronization strength between those oscillations - total percent of phase synchronization, measured in percent [9]. This index has already demonstrated its potential importance for clinical cardiology, for example, for evaluating of five-year fatal risk in patients after myocardial infarction (MI) and personalizing of medical therapy of coronary heart disease and hypertension [10-12]. 

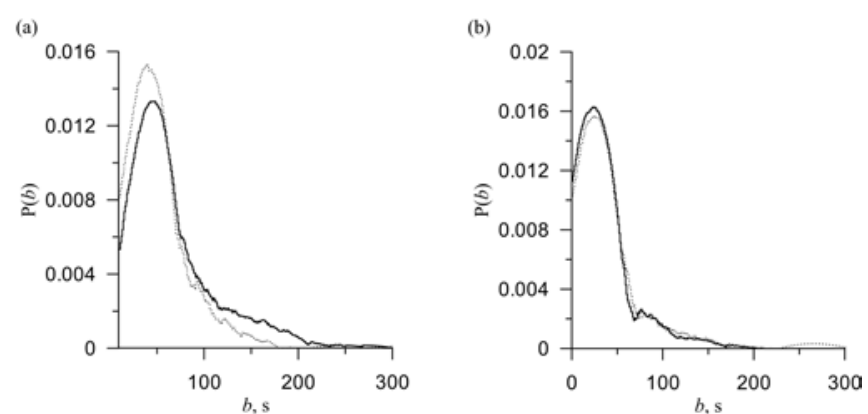

Figure 1. Sample probability density distribution $(P(b))$ for lengths of synchronous (a) and non-synchronous (b) sections of $0.1 \mathrm{~Hz}$ oscillations in HRV and PPGV from healthy subjects (solid line) and MI patients (dashed line).

However, total percent of phase synchronization only estimate the relation between time, which $0.1 \mathrm{~Hz}$ oscillations in HRV and PPGV were synchronized, and total length of observation (in our previous studies records were 10 minutes long). For healthy subjects this index varying in wide large of $20-60 \%$, however, statistically it is significantly different for patients with coronary heart disease, in particular, for those after MI [8]. Apparently this index is generalized measure of coupling strength between mechanisms of autonomic regulation of various parts of cardiovascular system, and is not suitable for studying of such characteristics of regulatory processes as: phase-synchronization sections longevity variation over time, asynchronous behavior sections longevity variation over time, extent of desynchronization, significance of the external factors influence on synchronization. Development of special criteria for detailed study of interactions between low-frequency oscillations in circulatory system is seems to be perspective for fundamental and clinical cardiology.

Groups with sufficiently opposing state of cardiovascular autonomic control, such as healthy young subjects and patients after $\mathrm{MI}$, can be used to investigate the potential possibility of detailed analysis of dynamical properties of interactions between $0.1 \mathrm{~Hz}$ oscillations in HRV and PPGV.

Objective of this article is to compare statistical characteristics of interaction between low-frequency oscillations in HRV and PPGV for healthy subjects and MI patients.

\section{Material and Methods}

We studied the following two groups of patients:

- 23 healthy subjects (20 men and 3 women) aged $26 \pm 3$ years;

- 23 patients (12 men and 11 women) aged $52 \pm 4$ years, one month after MI. Patients were undergoing hospital treatment in hospital of Research Institute of Cardiology (Saratov, Russia).

Electrocardiogram (ECG) and left ring finger photoplethysmogram (PPG) were simultaneously measured for each resting patient in supine position with $250 \mathrm{~Hz}$ sampling rate and 14 bit resolution. Signal of cardiointervalogram (CIG) sequence of time intervals between two consequent $R$ spikes was extracted from ECG signal. Equidistant CIG was then synthesized by interpolation of non-equidistant signal with cubicsplines with $5 \mathrm{~Hz}$ sample rate.
Rhythms that reflect activity of studied regulatory systems were then extracted by filtering CIG and PPG signals with band pass filter in the range of $[0.06,0.14] \mathrm{Hz}$. Sample rate of extracted PPG signal was limited by $5 \mathrm{~Hz}$ by decimation. Hilbert transformation was used to estimate instantaneous phases of oscillations of studied systems, and then the difference between estimated phases was calculated.

Borders of phase synchronization intervals and probabilitydensity functions (PDF) of lengths of synchronous and nonsynchronous sections were then determined for healthy subjects and $\mathrm{MI}$ patients via original method, proposed in our previous paper [9]. Total percent of phase synchronization was also calculated via method, proposed in [9]. PDF of slope angels of lines approximating the non-synchronous sections was used to estimate the variability of main frequency in low-frequency band of oscillations in HRV and PPGV. For both groups of subjects, elevation rate of instantaneous phase's difference was determined by the distribution of difference between instantaneous phases, which was also determined.

Nature and properties of phase noise, which is mainly determined by internal dynamical noises [13-15] but also includes measurement noises, is an important question that rises during studying of phase dynamics of systems of biological origin. Dispersion of phase noises was studied for $0.1 \mathrm{~Hz}$ oscillations in HRV and PPGV to compare the intensity of total influences of external and internal factors on interaction of low-frequency mechanisms of cardiovascular autonomic control. We used the special technic to extract phase noise. Phase noise was considered to be a residual of the sliding mean model for signal. Trends that were approximated via sliding mean model with $20 \mathrm{~s}$ length window ( 2 characteristic periods) were than subtracted from experimental phase's difference to estimate the noise characteristics. Statistical and spectral properties of the residuals of such models were estimated in this paper.

\section{Results}

Value of total percent of phase synchronization between $0.1 \mathrm{~Hz}$ oscillations in HRV and PPGV was $47 \pm 3 \%$ for healthy subjects and $26 \pm 4 \%$ for patients after MI (data are represented in the form of mean and standard deviation $-M \pm S D$ ).

Analysis of phase dynamics was used for detailed study of characteristics of interactions between low-frequency oscillations in HRV and PPGV. For both groups of subjects selective density functions were calculated for lengths of synchronous (Figure 1a) and non-synchronous (Figure $1 \mathrm{~b}$ ) sections of the records from the position of interaction between $0.1 \mathrm{~Hz}$ oscillations in HRV and PPGV. It appears that relatively short intervals of synchronization (about 30-70 seconds) interchanging with non-synchronous intervals of comparable length are prevailing for both groups. Significant difference was not detected between lengths distributions of synchronous and non-synchronous intervals calculated for studied low-frequency oscillations in healthy subjects and $\mathrm{MI}$ patients.

Figure 2 illustrate the results of estimation of variability of the main frequency in low-frequency band of oscillations in HRV and PPGV via calculation of PDF of slope angels of lines approximating the non-synchronous sections. Graphs represented in Figure 2 describe selective distributions of difference of instantaneous phases of studied oscillations in HRV and PPGV. For example, if instantaneous frequency of studied oscillation is equal to $0.105 \mathrm{~Hz}$ 
for HRV and $0.100 \mathrm{~Hz}$ for PPGV, than instantaneous phases difference (plotted on $X$ axis in Figure 2) is equal to $0.005 \mathrm{~Hz}$. During the whole record of ECG and PPG signals this difference is varying in certain range. If instantaneous phases of both oscillations are equal then difference will equal to zero. It appears that high degree of mismatches between main frequencies of lowfrequency oscillations in HRV and PPGV is typical for MI patients and not for healthy subjects.

Figure 3 represents the comparison between intensities of total influence that unaccounted external and internal factors had on interaction between low-frequency mechanisms of cardiovascular autonomic control. As it is seen from Figure 3 the phase noises distributions is close to normal. Mean value of phase noise dispersion (it was measured as mean level of dispersion between all subjects) is equal to $0.040 \pm 0.002$ for healthy subjects (Figure 3a) and $0.070 \pm 0.004$ (data are represented in the form of mean and standard error of the mean $-\mathrm{M} \pm \mathrm{m}$ ) for $\mathrm{Ml}$ patients (Figure $3 b$ ). From these results we concluded that intensity of phase noise is almost 2 times higher for diseased patients than for healthy ones. Results of the spectral analysis of the phase noises is represented in Figure 4. Maximum values of these spectra can be seen near $0.005 \mathrm{~Hz}$ and can represent the fluctuations associated with humoral regulation processes.

\section{Discussion}

Better understanding of characteristics of physiological processes that take place in cardiovascular system can be achieved by studying the properties of oscillation processes in this system. Low-frequency oscillations in circulation are known to be spontaneous and can be modulated [2]. Such oscillations in blood pressure are supposed to be of baroreflectory origin $[4,5,16]$, while origin of $0.1 \mathrm{~Hz}$ oscillations in PPGV are still matter of debate. It is crucial to consider during analysis of PPG and laser Doppler flowmetry (LDF) data. $0.1 \mathrm{~Hz}$ oscillations in PPG signal mainly characterize baroreflectory regulation of blood pressure, since dactylar arteries blood filling makes considerable contribution to this signal $[17,18]$. Whereas low-frequency oscillations in LDF are mainly characterize spontaneous vasomotor activity [19]. It is reasonable to consider cardiovascular autonomic control to be a complex nonlinear system with several self-exciting loops, because of functional independence between $0.1 \mathrm{~Hz}$ oscillations in HRV and blood pressure variability (and PPG) $[7,20]$ and ability of those rhythms to synchronize $[8,20]$. However, in spite of apparent reasons to analyze synchronization between $0.1 \mathrm{~Hz}$ oscillations [10-12], characteristics of this phenomenon are not yet studied.

Our study has revealed new information about modulation of main frequencies in low-frequency mechanisms of autonomic regulation by various factors that were treated as phase noise. It was shown that depth of such modulation can be associated with the quality of interaction between mechanisms of autonomic control. In particular, MI patients have significantly higher phase noise in $0.1 \mathrm{~Hz}$ oscillations in HRV and PPGV, than healthy subjects. This fact can be due to either heighten senility of the autonomic regulation to the external factors, or due to cardiovascular disease pathogenesis and its strong influence. Heighten variability of the main rhythm in low-frequency band can also be due to overall functional instability of the regulatory mechanism in respect to external factors.

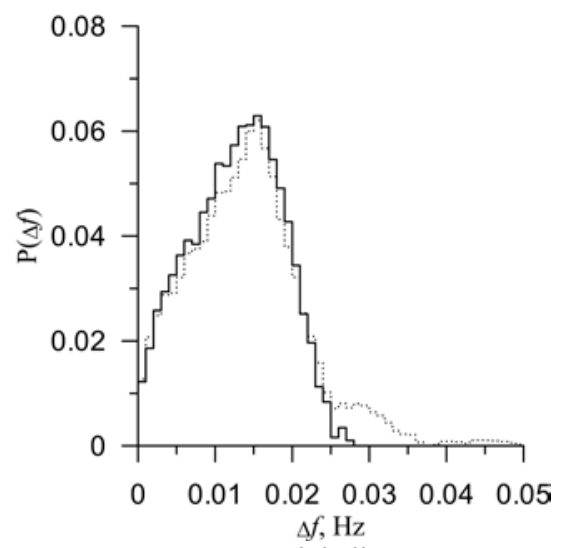

Figure 2. Distribution histogram $(P(\Delta f))$ of instantaneous phases difference $(\Delta f)$ between studied loops of autonomic regulation in healthy subjects (solid line) and MI patients (dashed line).
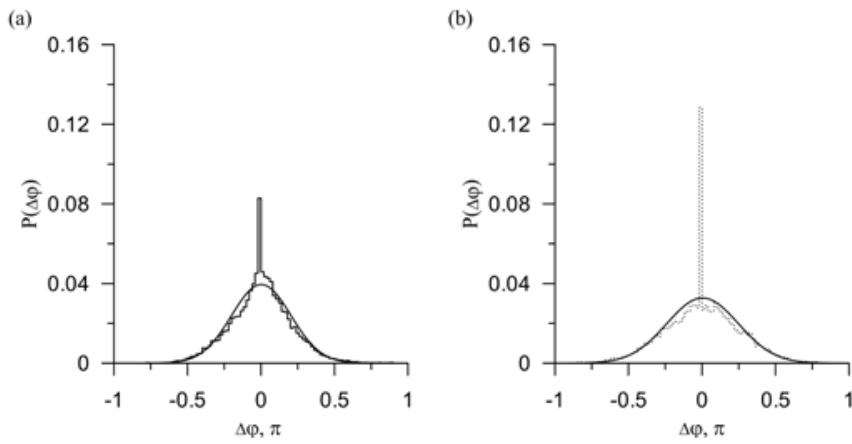

Figure 3. Phase noise distribution for a set of healthy subjects (a) and M patients (b) approximated by Gaussian distribution.

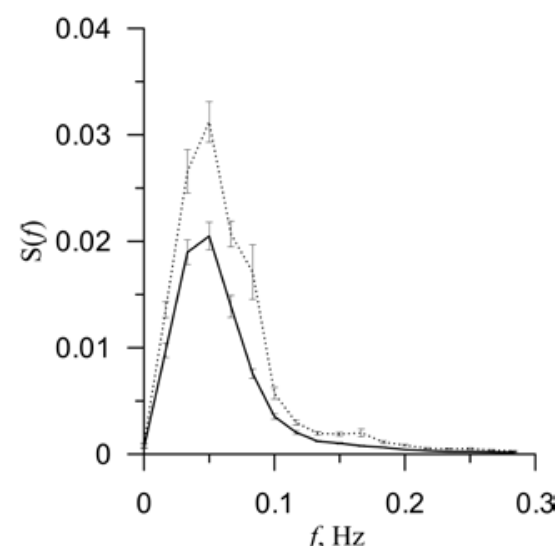

Figure 4. Estimation and error of the mean of spectral power density of phase noise calculated for set of healthy subjects (solid line) and MI patients (dashed line).

Although lengths of synchronous sections are almost equal (difference of about several seconds) for healthy subjects and $\mathrm{MI}$ patients, total time of desynchronization between $0.1 \mathrm{~Hz}$ oscillations is considerably larger for MI patients. 


\section{Conclusion}

It was shown that relatively short interchangeable intervals of synchronous and non-synchronous behavior are typical for $0.1 \mathrm{~Hz}$ oscillations in HRV and PPGV for both healthy subjects and $\mathrm{MI}$ patients, and therefore can characterize interaction between lowfrequency mechanisms of cardiovascular autonomic control. However, internal and external factors seem to have higher influence on $\mathrm{Ml}$ patients system of autonomic control of circulation. $\mathrm{MI}$ patients in comparison with healthy subjects have higher level of phase noise in $0.1 \mathrm{~Hz}$ oscillation in HRV and PPGV. Also in comparison with healthy subjects $\mathrm{MI}$ patients have higher degree of mismatch between main rhythms in low-frequency oscillations in HRV and PPGV.

\section{Conflict of interest: not declared.}

\section{Acknowledgment}

This work was supported by the President of the Russian Federation (Grant No. MD-4368.2015.7) and the Russian Foundation for Basic Research (Grant No. 15-02-03061, and Grant No. 16-32-00326).

\section{References}

1. Heart rate variability: standards of measurement, physiological interpretation and clinical use. Task Force of the European Society of Cardiology and the North American Society of Pacing and Electrophysiology. Circulation 1996; 93(5): 1043-1065. (PMID: 8598068) (doi: 10.1161/01.CIR.93.5.1043).

2. Obrig $H$, Neufang $M$, Wenzel $R$, Kohl $M$, Steinbrink J, Einhäupl K, Villringer A. Spontaneous low frequency oscillations of cerebral hemodynamics and metabolism in human adults. Neuroimage 2000; 12: 623-639. (PMID: 11112395) (doi: 10.1006/nimg.2000.0657)

3. Song S, Kim D, Jang DP, Lee J, Lee H, Lee KM, Kim IY. Low-frequency oscillations in cerebrovascular and cardiovascular hemodynamics: Their interrelationships and the effect of age. Microvasc Res 2015; 102: 46-53. (doi: 10.1016/j.mvr.2015.08.004) (PMID: 26277229)

4. Burgess DE, Hundley JC, Li SG, Randall DC, Brown DR. First-order differential-delay equation for the baroreflex predicts the $0.4-\mathrm{Hz}$ blood pressure rhythm in rats. Am J Physiol 1997; 273: R1878-R1884. (PMID: 9435640)

5. Ringwood JV, Malpas SC. Slow oscillations in blood pressure via a nonlinear feedback model. Am J Physiol Regul Integr Comp Physiol 2001; 280(4): R1105-R1115. (PMID: 11247833)

6. Borovkova YI, Karavaev AS, Bezruchko BP, Ponomarenko VI, Prokhorov MD. Uncovering frequency locking for systems affected by chirping. Bulletin of the Russian Academy of Sciences: Physics 2011; 75(12): 1601-1604. (doi: 10.3103/S1062873811120057)

7. Karavaev AS, Kiselev AR, Gridnev VI, Borovkova EI, Prokhorov MD, Posnenkova OM, et al. Phase and frequency locking of $0.1-\mathrm{Hz}$ oscillations in heart rate and baroreflex control of blood pressure by breathing of linearly varying frequency as determined in healthy subjects. Human Physiology 2013; 39(4): 416-425. (doi: 10.7868/S0131164613010049)

8. Kiselev AR, Bespyatov AB, Posnenkova OM, Gridnev VI, Ponomarenko VI, Prokhorov MD, Dovgalevskii PYa. Internal synchronization of the main $0.1-\mathrm{Hz}$ rhythms in the autonomic control of the cardiovascular system. Human Physiology 2007; 33(2): 188-193. (doi: 10.1134/S0362119707020089)

9. Kiselev AR, Karavaev AS, Gridnev VI, Prokhorov MD, Ponomarenko VI, Borovkova El, et al. Method of estimation of synchronization strength between low-frequency oscillations in heart rate variability and photoplethysmographic waveform variability. Russian Open Medical Journal 2016; 5: e0101. (doi: 10.15275/rusomj.2016.0101)
10. Kiselev AR, Gridnev VI, Prokhorov MD, Karavaev AS, Posnenkova OM, Ponomarenko VI, Bezruchko BP. Selection of optimal dose of betablocker treatment in myocardial infarction patients based on changes in synchronization between $0.1 \mathrm{~Hz}$ oscillations in heart rate and peripheral microcirculation. Journal of Cardiovascular Medicine 2012; 13(8): 491-498. (doi: 10.2459/JCM.0b013e3283512199) (PMID: 22343262)

11. Kiselev AR, Gridnev VI, Prokhorov MD, Karavaev AS, Posnenkova OM, Ponomarenko $\mathrm{VI}$, et al. Evaluation of 5-year risk of cardiovascular events in patients after acute myocardial infarction using synchronization of $0.1-\mathrm{Hz}$ rhythms in cardiovascular system. Ann Noninvasive Electrocardiol 2012; 17(3): 204-213. (doi: 10.1111/j.1542474X.2012.00514.x) (PMID: 22816539)

12. Kiselev AR, Gridnev VI, Prokhorov MD, Karavaev AS, Posnenkova OM, Ponomarenko VI, Bezruchko BP. Effects of antihypertensive treatment on cardiovascular autonomic control: a prospective study. Anadolu Kardiyol Derg 2014; 14: 701-710. (doi: 10.5152/akd.2014.5107) (PMID: 25188759)

13. Bunde A, Havlin S, Kantelhardt JV, Penzel T, Peter JH, Voigt K. Correlated and uncorrelated regions in heart-rate fluctuations during sleep. Phys Rev Lett 2000; 85: 3736-3739. (PMID: 11030994) (doi: 10.1103/PhysRevLett.85.3736)

14. Togo F, Yamamoto Y. Decreased fractal component of human heart rate variability during non-REM sleep. Am J Physiol Heart Circ Physiol 2001; 280(1): H17-H21. (PMID: 11123213)

15. Kotani K, Struzik ZR Takamasu K, Stanley HE, Yamamoto Y. Model for complex heart rate dynamics in health and disease. Physical Review $E$ 2005; 72: 041904. (doi: 10.1103/PhysRevE.72.041904)

16. Horsman HM, Peebles KC, Tzeng YC. Interactions between breathing rate and low-frequency fluctuations in blood pressure and cardiac intervals. J Appl Physiol 2015; 119(7): 793-798. (doi: 10.1152/japplphysiol.00525.2015) (PMID: 26205543)

17. Higgins JL, Fronek A. Photoplethysmographic evaluation of the relationship between skin reflectance and skin blood volume. J Biomed Engineering 1986; 8: 130-136. (PMID: 3713144)

18. Rhee $\mathrm{S}$, Yang $\mathrm{BH}$, Asada $\mathrm{H}$. Theoretical evaluation of the influence of displacement on finger photoplethysmography for wearable health monitoring sensors. In: ASME International Mechanical Engineering Congress and Exposition, Symposium on Dynamics, Control, and Design of Biomechanical Systems. Nashville, Tennessee, November 14-19, 1999.

19. Stefanovska A, Bracic M, Kvernmo HD. Wavelet analysis of oscillations in the peripheral blood circulation measured by laser Doppler technique. IEEE Trans Biomed Eng 1999; 46: 1230-1239. (PMID: 10513128) (di: 10.1109/10.790500)

20. Guasti L, Mainardi LT, Baselli G, Simoni C, Cimpanelli M, Braga SS, et al. Components of arterial systolic pressure and RR-interval oscillation spectra in a case of baroreflex failure, a human open-loop model of vascular control. J Hum Hypertens 2010; 24(6): 417-426. (doi: 10.1038/jhh.2009.79) (PMID: 19907435)

\section{Authors:}

Vladimir A. Shvartz - MD, PhD, Researcher, Department of Surgical Treatment for Interactive Pathology, Bakulev Scientific Center for Cardiovascular Surgery, Moscow, Russia.

Anatoly S. Karavaev - PhD, Associate Professor, Department of Nano- and Biomedical Technologies, Saratov State University n.a. N.G. Chernyshevsky, Saratov, Russia.

Ekaterina I. Borovkova - MSc, PhD student, Department of Nano- and Biomedical Technologies, Saratov State University n.a. N.G. Chernyshevsky, Saratov, Russia.

Sergey A. Mironov - MD, PhD, Physician, Central Clinical Military Hospital, Moscow, Russia.

Vladimir I. Ponomarenko - DSC, Professor, Department of Nano- and Biomedical Technologies, Saratov State University n.a. N.G. Chernyshevsky, Saratov, Russia; Leading Researcher, Saratov Branch of the Institute of 
Radio Engineering and Electronics of Russian Academy of Sciences, Saratov, Russia.

Mikhail D. Prokhorov - DSc, Head of Laboratory, Saratov Branch of the Institute of Radio Engineering and Electronics of Russian Academy of Sciences, Saratov, Russia.

Yurii M. Ishbulatov - BSc, Postgraduate student, Department of Nano- and Biomedical Technologies, Saratov State University n.a. N.G. Chernyshevsky, Saratov, Russia.

Elena E. Lapsheva - Lecturer, Department of Computer Sience and IT, Saratov State University n.a. N.G. Chernyshevsky, Saratov, Russia.

Vladimir I. Gridnev - MD, DSc, Head of Department of New Cardiological Informational Technologies, Research Institute of Cardiology, Saratov State Medical University n.a. V.I. Razumovsky, Saratov, Russia; Professor, Department of Nano- and Biomedical Technologies, Saratov State University n.a. N.G. Chernyshevsky, Saratov, Russia.

Anton R. Kiselev - MD, DSc, Leading Researcher, Department of New Cardiological Informational Technologies, Research Institute of Cardiology, Saratov State Medical University n.a. V.I. Razumovsky, Saratov, Russia; Professor, Department of Nano- and Biomedical Technologies, Saratov State University n.a. N.G. Chernyshevsky, Saratov, Russia; Researcher, Department of Surgical Treatment for Interactive Pathology, Bakulev Scientific Center for Cardiovascular Surgery, Moscow, Russia. 